\title{
Receptor-mediated inhibitory mechanisms and the regulation of platelet function
}

\author{
CHENG ZhiPeng ${ }^{1,2} \&$ LIU JunLing ${ }^{1 *}$ \\ ${ }^{1}$ Department of Biochemistry and Molecular Cell Biology, Shanghai Jiao Tong University School of Medicine, Shanghai 200025, China; \\ ${ }^{2}$ Institute of Hematology, Union Hospital, Tongji Medical College, Huazhong University of Science and Technology, Wuhan 430074, China
}

Received August 22, 2015; accepted September 11, 2015; published online November 19, 2015

Citation: Cheng ZP, Liu JL. Receptor-mediated inhibitory mechanisms and the regulation of platelet function. Sci China Life Sci, 2015, 58: 1299-1301, doi: 10.1007/s11427-015-4966-5

Platelets are derived from megakaryocytes; they circulate in mammalian blood vessels and play essential roles in hemostasis and thrombosis. When platelets encounter injury, they respond by triggering the formation of a blood clot to prevent blood loss (hemostasis). While this is vital for survival, platelets can cause blood clots inside arteries (known as thrombosis), which can trigger heart attack and stroke. Platelets express a variety of receptors, including adhesive receptors, such as glycoprotein (GP) IIb-IIIa ( $\alpha \mathrm{IIb} \beta 3)$, GPIb-IX-V, GPIa-IIa $(\alpha 2 \beta 1)$, and GPVI, which can mediate the adherence of platelets to exposed collagen fibers at the sites of blood vessel injury or atherosclerosis plaque rupture [1]. These adhesive receptors initiate intracellular signals that cause platelet adhesion, spreading, and secretion. The activated platelets release several soluble agonists, such as adenosine diphosphate (ADP), prostanoid thromboxane A2 (TXA2), and thrombin. These agonists amplify the initial signals and recruit additional platelets from the circulation into a growing thrombus. The amplification of platelet activation and recruitment is mediated by $\mathrm{G}$ protein coupled receptors (GPCRs), such as the ADP receptors P2Y1 and P2Y12 (coupled to Gq and Gi, respectively), the TXA2 receptor Tp (coupled to Gq and G12/13) and PARs, which bind thrombin (coupled to Gi, Gq and G12/13) [2]. The functions and downstream signals of these platelet adhesive receptors in hemostasis and thrombosis have been well characterized. However, the currently most widely

*Corresponding author (email: liuj1@ shsmu.edu.cn) used anti-platelet drugs, such as the antagonists of $\alpha \operatorname{IIb} \beta 3$ and P2Y12, target on the activation mechanisms and have low efficacy in many patients and are associated with serious bleeding side-effects. To develop more effective antithrombotic drugs, understanding how platelet activation is regulated is necessary.

The accumulated evidence over the past decade indicates that endogenous receptor-mediated inhibitory mechanisms keep platelets quiescent and prevent excessive platelet activation. In this review, we discuss the mechanisms and therapeutic potential of inhibitory pathways in the regulation of platelet activation.

\section{Inhibitory immunoglobulin superfamily (IgSF)}

IgSF proteins have been reported to be expressed on the plasma membranes of platelet and to regulate platelet adhesion, aggregation and secretion. Among the IgSFs, platelet collagen receptor GPVI initiates platelet activating signals by binding to the immunoreceptor tyrosine-based activation motif (ITAM) of the Fc receptor $\gamma$-chain (FcR $\gamma$-chain) when platelets become tethered to the collagen and laminin proteins of the exposed subendothelial matrix. The GPVI/ FcR $\gamma$-chain complex disseminates powerful signals, causing $\alpha \operatorname{IIb} \beta 3$ activation and platelet secretion and aggregation. In addition, IgSFs also include C-type lectin-like receptor 2 
(CLEC-2), which mediate hemITAM signaling [3].

In addition to the activation mechanisms mentioned above, platelets also express IgSF inhibitory receptors, such as platelet endothelial cell adhesion molecule-1 (PECAM-1), G6B, and carcinoembryonic antigen-related cell adhesion molecule 1 and 2 (CEACAM1 and 2) [4]. Their cytoplasmic domains bearing immunoreceptor tyrosine-based inhibitory motifs (ITIMs) have the capacity to bind multiple phosphatases, including SHP-1 and SHP-2, to attenuate cell signals; thus, they negatively regulate GPVI/FcR $\gamma$ chain and CLEC-2-mediated platelet activation. In addition to the phosphatases SHP-1 and SHP-2, our study revealed that phosphatase and tensin homolog (PTEN), a negative regulator of phosphoinositide 3-kinase (PI3K) pathways, inhibits GPVI/FcR $\gamma$-chain-mediated platelet activation via its inositol phospholipid phosphatase and tyrosine phosphatase functions [5].

Our group also recently found that ITIMs bearing paired immunoglobulin-like receptor B (PIRB) and its human ortholog leukocyte immunoglobulin-like receptor B2 (LILRB2) were highly expressed in mouse and human platelets, respectively [6]. Deletion of the PIRB intracellular domain significantly facilitates agonist-induced mouse platelet aggregation and platelet spreading on immobilized fibrinogen. Characterization of the signaling involved in this process indicated that PIRB associates with tyrosine phosphatases SHP1/2 and attenuates tyrosine phosphorylation of the linker for activation of $\mathrm{T}$ cells (LAT) signalosome, integrin $\beta 3$ and its downstream focal adhesion kinase (FAK) to inhibit platelet activation. Of particular interest, we found that PIRB/LILRB2 potential ligand angiopoietin-likeprotein 2 (ANGPTL2) is expressed and stored in platelet $\alpha$-granules and is released upon platelet activation to inhibit agonist-induced platelet activation [6]. Therefore, our study reveals a new self-control mechanism for the negative regulation of platelet activation and a new antithrombotic therapeutic target, the ANGPTL2/PIRB pathway [7].

\section{Inhibitory nuclear receptors}

Recently, several intracellular receptors, such as the liver X receptor (LXR), retinoid $\mathrm{X}$ receptor (RXR), peroxisome proliferator activated receptor $\gamma(\operatorname{PPAR} \gamma)$ and glucocorticoid receptor (GR) [8], have been shown to be expressed in platelets. Although intracellular receptors in anucleated platelets seem unnecessary, agonists to RXR, LXR, PPAR $\gamma$ and GR significantly interfere with platelet function in a nongenetic manner.

Retinoid X receptors (RXRs) are important transcriptional nuclear hormone receptors. Human platelets express RXR $\alpha$ and RXR $\beta$. RXR ligands exert an inhibitory effect on platelet aggregation mediated by ADP and the TXA2 receptors, but only weakly on that by collagen receptor. An
RXR can bind to Gq, thereby, inhibit Gq coupled receptor-induced Rac activation and intracellular calcium release.

The peroxisome proliferator-activated receptors (PPARs) consist three nuclear receptor isoforms $(\alpha, \beta / \delta$, and $\gamma)$ and associates with the retinoic $\mathrm{X}$ receptor (RXR). PPAR $\gamma$ is expressed in human platelets, and its ligands significantly inhibit platelet aggregation in vitro and thrombus formation in vivo. After platelet activation, PPAR $\gamma$ associates with Syk and LAT, which are downstream signaling molecules of the collagen receptor GPVI, indicating a potential mechanism of PPAR $\gamma$ in the negative regulation of GPVI-mediated signaling. Of particular interest statins, which are cholesterol-lowering drugs, inhibit platelet activation by engaging PPAR $\alpha$ and PPAR $\gamma$.

Liver X receptors (LXRs) are transcription factors that are involved in the regulation of cholesterol homeostasis. Dr. Gibbin's group [8] recently showed that LXR- $\beta$ is present in human platelets and that the LXR ligands GW3965 and T0901317 suppress platelet aggregation stimulated by agonists. Mechanism analysis revealed that active LXR was able to associate with signaling components of the collagen receptor GPVI, resulting in an inhibition of platelet activation.

Glucocorticoid receptor (GR) was also identified in human platelets, and the GR ligand prednisolone extensively reduces $\mathrm{ADP}$ and TXA2 receptor-mediated platelet aggregation. The antithrombotic effects of nuclear receptor ligands further indicate that nuclear receptors are potential targets for the prevention of athero-thrombotic disease.

\section{Inhibitory prostanoid receptors}

Prostanoids are a family of metabolic derivatives of a saturated fatty acid or prostanoic acid and play critical roles in the platelet-vascular wall interaction. Several enzymes, such as phospholipase A2, cyclooxygenases (COXs) and tissue specific enzymes, are involved in the synthesis of prostanoids. There are a variety of prostanoids, such as thromboxane A2 (TXA2), prostaglandin E2 (PGE2), prostacyclin (PGI2), and prostaglandin D [9].

TXA2, the major prostanoid product in platelets, is a potent stimulator of platelet aggregation via activation of a TXA2-specific GPCR receptor (TP). In atherosclerotic plaques, prostaglandin $\mathrm{H} 2$ ( $\mathrm{PGH} 2)$ is preferentially converted into PGE2 by active macrophages. Macrophage-derived PGE2 can be released to activate EP3 receptors on platelets, which exacerbates arterial thrombosis. Aspirin irreversibly inhibits platelet cyclooxygenase 1 preventing the formation of PGH2 and, therefore, TXA2.

In contrast, PGI2, the major prostanoid product of the macrovascular endothelium, has an intensively inhibitory function via the modulation of platelet intracellular cyclic nucleotide levels [10]. PGI2 binds to its Gs coupled receptor, IP, and elevates the intracellular levels of cAMP. As a 
result, the activity of protein kinase A (PKA) is elevated, which inhibits platelet activation by directly suppressing activation signals mediated by Gi coupled receptors, such as P2Y12, TP and the PGE2 receptor EP3. Similarly, the prostaglandin D receptor DP1 and the PGE2 receptors EP2 and EP4 are coupled to Gas and are highly expressed in platelets. Activation of DP1, EP2 and EP4 results in inhibition of platelet activation.

The reciprocal regulation of platelet function by endogenous prostanoids and their activating and inhibitory prostanoid receptors brings new insight into the regulation of platelet functions. And high selective ligands of inhibitory prostanoid receptors can be developed as a new class of antiplatelet reagents.

In summary, there naturally exist a variety of inhibitory receptors, which are critical for the regulation of platelet activation. Functional cross-talk between endogenous activating and inhibitory mechanisms generate networks that maintain platelet homeostasis. The most commonly used anti-platelet drugs targeting activation mechanism have low efficacies in many patients and cause serious bleeding side-effects. Excitation of endogenous inhibitory mechanism in platelets may bring hope to the development of more reliable therapeutic approaches to thrombotic diseases.

The author(s) declare that they have no conflict of interest.

This work was supported by the National Natural Science Foundation of
China (91439115) and the National Basic Research Program of China (NO2012CB518000).

1 Davi G, Patrono C. Platelet activation and atherothrombosis. N Eng J Med, 2007, 357: 2482-2494

2 Offermanns S. Activation of platelet function through $\mathrm{G}$ protein-coupled receptors. Circ Res, 2006, 99: 1293-1304

3 Moroi AJ, Watson SP. Impact of the PI3-kinase/Akt pathway on ITAM and hemITAM receptors: haemostasis, platelet activation and antithrombotic therapy. Biochem Pharmacol, 2015, 94: 186-194

4 Alshahrani MM, Yang E, Yip J, Ghanem SS, Abdallah SL, deAngelis AM, O'Malley CJ, Moheimani F, Najjar SM, Jackson DE. CEACAM2 negatively regulates hemi (ITAM-bearing) GPVI and CLEC-2 pathways and thrombus growth in vitro and in vivo. Blood, 2014, 124: 2431-2441

5 Weng Z, Li D, Zhang L, Chen J, Ruan C, Chen G, Gartner TK, Liu J. PTEN regulates collagen-induced platelet activation. Blood, 2010, 116: 2579-2581

6 Fan X, Shi P, Dai J, Lu Y, Chen X, Liu X, Zhang K, Wu X, Sun Y, Wang K, Zhu L, Zhang CC, Zhang J, Chen GQ, Zheng J, Liu J. Paired immunoglobulin-like receptor B regulates platelet activation. Blood, 2014, 124: 2421-2430

7 Du X. Self-control of platelets: a new ITIM story. Blood, 2014, 124: 2322-2323

8 Spyridon M, Moraes LA, Jones CI, Sage T, Sasikumar P, Bucci G, Gibbins JM. LXR as a novel antithrombotic target. Blood, 2011, 117: 5751-5761

9 Leslie CC. Cytosolic phospholipase A2: physiological function and role in disease. J Lipid Res, 2015, 56: 1386-1402

10 Yu Y, Ricciotti E, Scalia R, Tang SY, Grant G, Yu Z, Landesberg G, Crichton I, Wu W, Puré E, Funk CD, FitzGerald GA. Vascular COX-2 modulates blood pressure and thrombosis in mice. Sci Transl Med, 2012, 4: 132ra154

Open Access This article is distributed under the terms of the Creative Commons Attribution License which permits any use, distribution, and reproduction in any medium, provided the original author(s) and source are credited. 